\title{
Research on Prediction of Different Categories of Video based on YOUTUBE Using Text Mining and Sentiment Analysis
}

\author{
Lin Gan * \\ Department of Computing, the Hong Kong Polytechnic University, Hong Kong, China \\ * missmissganlin@gmail.com
}

\begin{abstract}
With the development of online commentary research, scholars have tried to tap into the deeper value of online commentary from the analysis of sentiment analysis, quality evaluation, false comment recognition to the usefulness of comments. Previous studies have focused on online product reviews while news reviews. Social media research has been relatively rare. social media and news commentary contain readers' opinions and evaluations on current events, and reflect the trend of public opinion. The purpose of this paper is to investigate and analyze the intrinsic link between social media content of different type and the number of commentaries, and sentiment analysis.
\end{abstract}

Keywords: Social Media; Video; Text Mining; Sentiment Analysis.

\section{Introduction}

There are many social networks in use today, like Facebook, Twitter, YouTube, and many others. Social media has been broadly used in the recommender system, sentiment analysis, text mining, business approach and marketing. A key aspect of social networks is richer in information that brings the challenges and opportunities in the data mining and knowledge discovery. There makes it appear that many researchers have started to study online reviews and have already made good progress. For example, according to the usefulness of online product reviews, the reviews can be sorted and then followers Can get comments with more valuable information, which makes easier to complete next step decisions. Further, most social media researches focused on excavating the emotional factors contained in commentary, evaluating the quality of different content commentary.

\section{Literature Review}

In 1999, Leets et al. (1995) explored the reasons why people sent letters to celebrities. Nowadays, a lot of people and media join the social media platform and frequently interact with their followers. In this study, we aim to investigate the underlying reasons of people leaving their comments on different channels of videos. According to comments based on Leets et al. (1995)'s categorization of why people Interact with celebrities. we got an idea that collected different YouTube sample comments. This helps us to explore this kind of comments at a larger scale. The collected data will be summarized and discussed. The following hypotheses are thus drawn accordingly. Are the number of comments on social media directly related to video contents?

$\mathrm{H}_{1}$, Whether the categories of videos affect the number of comments on social media?

$\mathrm{H}_{2}$, Whether the viewer's comments directly related to the videos content? (positive/negative).

(This paper extracts text features, sentiment features, data analysis from different type videos reviews according to relevant research, formulates an evaluation system for video review and video categories popularity prediction task.).

\section{Methods}

We collected six topics videos on the YouTube hot search list from February 31. January 2019 to.

The first step is choosing the top 6 popular categories of videos on YouTube, like Haul video, Online teaching, VLOGs, Game video, Comedy /Skit video and Unboxing Videos. 
The second Step is collecting each category 8-10 samples in same categories of channel. Moreover, each channel choose6 equally number of viewers for comparison.

Table 1. Online teaching INCLUDING Beauty (makeup) \& Cooking

\begin{tabular}{|c|c|c|c|c|c|c|}
\hline $\begin{array}{c}\text { Channel } \\
\text { name }\end{array}$ & $\begin{array}{l}\text { More Than } \\
15 \mathrm{M} \text { views }\end{array}$ & $5 \mathrm{M}$ viwes & $\begin{array}{l}\text { More than } 1 \mathrm{M} \\
\text { views }\end{array}$ & 900Kviews & $300 \mathrm{~K}$ views & $100 \mathrm{~K}$ views \\
\hline $\begin{array}{l}\text { Bethany } \\
\text { Mota }\end{array}$ & $\begin{array}{l}\mathrm{v}=\text { koMbIaJ } \\
\text { 8Tmo } \\
\text { Morning } \\
\text { Routine: } \\
\text { Fall } \\
\text { Edition!! }\end{array}$ & $\begin{array}{c}\mathrm{v}=\text { EoakbXJmc } \\
\text { to } \\
\text { Fears, TV } \\
\text { Shows, } \\
\text { Boyfriend? } \\
\text { TMI TAG! }\end{array}$ & $\begin{array}{c}\mathrm{v}=0 \text { K5XdYe4E } \\
\text { GA } \\
\text { name: } \\
\text { Instagram } \\
\text { Followers } \\
\text { Control My } \\
\text { Life for a Day | } \\
\text { Bethany Mota } \\
1693\end{array}$ & $\begin{array}{c}\text { v=PbH8oC_Oy } \\
\text { VM } \\
\text { Name: Summer } \\
\text { Try-On } \\
\text { Clothing Haul! } \\
\text { Forever 21, } \\
\text { Urban } \\
\text { Outfitters, \& } \\
\text { more! }\end{array}$ & 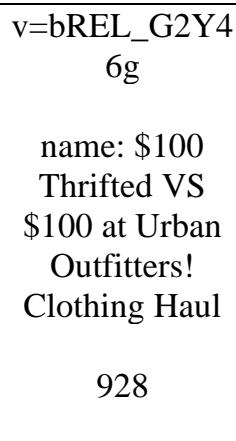 & $\begin{array}{c}\text { v=I1qczcjh9X } \\
\text { M } \\
\text { name: Spring } \\
\text { Jewelry - My } \\
\text { Must Have } \\
\text { Pieces! } \\
628\end{array}$ \\
\hline $\begin{array}{l}\text { Michelle } \\
\text { Phan }\end{array}$ & $\begin{array}{c}\mathrm{v}=\mathrm{qg}- \\
\text { 2rDnWCJA } \\
\\
\text { Seductive } \\
\text { Vampire }\end{array}$ & $\begin{array}{c}\mathrm{v}=\text { zupy7R4uR } \\
2 \mathrm{~g} \\
\text { Seductive } \\
\text { Smokey Eyes } \\
\text { Tutorial }\end{array}$ & $\begin{array}{c}\mathrm{v}=\text { MXm7obily } \\
\text { HM } \\
\text { Final Dynasty : } \\
\text { Aesthetic } \\
\text { Inspiration } \\
1713\end{array}$ & $\begin{array}{c}\mathrm{v}=\mathrm{sw} \text { crprNIPT } \\
\mathrm{c} \\
\text { Trend Report: } \\
\text { Glitter } \\
\text { Freckles, } \\
\text { Geometric } \\
\text { Undercuts + } \\
\text { more } \\
1214\end{array}$ & $\begin{array}{c}\mathrm{v}=\mathrm{cYSYnWwy} \\
\text { 3R8 } \\
\text { Pillow Talk 8: } \\
\text { Cyber-Bullying } \\
\text { and Acne } \\
\text { Shaming } \\
731\end{array}$ & N/A \\
\hline $\begin{array}{l}\text { Rachel } \\
\text { Levin }\end{array}$ & $\mathrm{N}$ & $\begin{array}{c}\text { v=eGhsWJgbu } \\
\text { zg } \\
\text { Doing My } \\
\text { Makeup In } \\
\text { 5000 Pounds } \\
\text { of Jello With } \\
\text { James Charles! }\end{array}$ & $\begin{array}{c}\text { v=18W_RpxDa } \\
\text { 3I } \\
\text { Swapping } \\
\text { Outfits With } \\
\text { Tana Mongeau! } \\
1964\end{array}$ & $\begin{array}{c}\mathrm{v}=\mathrm{XNxoeO} \_\mathrm{yU} \\
14 \\
\text { Christmas } \\
\text { Carpool Ride! } \\
2108\end{array}$ & $\begin{array}{c}\mathrm{v}=\mathrm{MhW} 8 \mathrm{pL} 2 \mathrm{jx} \\
10 \\
\text { Handcuffing } \\
\text { My Boyfriend } \\
\text { To Josh Peck } \\
\text { For A Day! } \\
1644\end{array}$ & $\begin{array}{c}\mathrm{v}=- \\
\text { _sw3o5Szcs } \\
\\
5 \text { Cute } \\
\text { Valentines } \\
\text { Nail Designs }\end{array}$ \\
\hline $\begin{array}{l}\text { Nikkie } \\
\text { De Jager }\end{array}$ & $\begin{array}{l}\mathrm{v}=\text { SOoauH } \\
\text { BpcbU } \\
\text { Side by Side } \\
\text { + Jessie J }\end{array}$ & $\begin{array}{c}\mathrm{v}=\mathrm{fUgxlywvV} \\
\text { Hc } \\
\text { How to } \\
\text { Contour \& } \\
\text { Highlight }\end{array}$ & $\begin{array}{c}\mathrm{v}=\text { CGJkvTjoV5 } \\
\text { Y } \\
\text { TRANSFORMI } \\
\text { NG MY MOM } \\
\text { INTO ME! } \\
2082\end{array}$ & $\begin{array}{c}\mathrm{v}=\mathrm{QMQROHtt} \\
\text { crc } \\
\text { Glittery Double } \\
\text { Cut Crease \& } \\
\text { Purple Lips } \\
\text { Holiday } \\
\text { Makeup } \\
1815\end{array}$ & $\begin{array}{c}\text { v=KmZZGfntd } \\
\text { Qw } \\
\text { SNAPCHAT } \\
\text { Q\&A: Huda } \\
\text { Beauty, Getting } \\
\text { Married \& } \\
\text { MORE! }\end{array}$ & $\begin{array}{c}\text { v=IpoUjFgc- } \\
\qquad 2 \mathrm{~g} \\
\text { Edgy Flapper } \\
\text { Girl Makeup } \\
\text { Tutorial · } \\
\text { RuPaul's Drag } \\
\text { Race Series } \\
272\end{array}$ \\
\hline $\begin{array}{l}\text { food } \\
\text { wishes }\end{array}$ & N/A & $\begin{array}{c}\text { v=uxEhH6MP } \\
\text { H28 } \\
\text { Buttermilk } \\
\text { Fried Chicken }\end{array}$ & $\begin{array}{c}\mathrm{v}=\text { UcqzDbB- } \\
9 \mathrm{tI} \\
\\
\text { Lemon Pepper } \\
\text { Potatoes - Food } \\
\text { Wishes } \\
\\
1117\end{array}$ & $\begin{array}{c}\mathrm{v}=71 \mathrm{ZJR} \text { shrEJ } \\
\mathrm{M} \\
\\
\text { Butcher's Steak } \\
\text { (aka Hanger } \\
\text { Steak) - How to } \\
\text { Trim and Cook } \\
\text { Butcher's Steak } \\
986\end{array}$ & $\begin{array}{c}\mathrm{v}=3 \mathrm{IZFd} 014 \mathrm{vQ} \\
\mathrm{M} \\
\text { German Potato } \\
\text { Dumplings } \\
\text { (Kartoffelkloes } \\
\text { se) - Food } \\
\text { Wishes } \\
1166\end{array}$ & $\begin{array}{c}\mathrm{v}=16 \mathrm{Q} 2 \mathrm{QMbE} \\
\text { gZk } \\
\text { Chef John's } \\
\text { White Bread - } \\
\text { Food Wishes } \\
726\end{array}$ \\
\hline
\end{tabular}




\begin{tabular}{|c|c|c|c|c|c|c|}
\hline Munchie & N/A & N/A & $\begin{array}{c}\mathrm{v}=\mathrm{eUPYFt} 52 \mathrm{~F} \\
\text { BE } \\
\text { How To Make } \\
\text { Jamaican Beef } \\
\text { Patties } \\
1296\end{array}$ & $\begin{array}{c}\mathrm{v}=0 \mathrm{fxL} 8 \mathrm{v} 2 \mathrm{dMh} \\
\text { o } \\
\text { How to } \\
\text { Sharpen a } \\
\text { Knife with a } \\
\text { Japanese } \\
\text { Master } \\
\text { Sharpener } \\
350\end{array}$ & $\begin{array}{c}\text { v=iGtqF3N6w } \\
\text { CI } \\
\text { The Capital of } \\
\text { Cecina Tacos - } \\
\text { Ultimate Taco } \\
\text { Tour of Mexico } \\
245\end{array}$ & $\begin{array}{c}\mathrm{v}=\mathrm{Q7OqC0qtJ} \\
\text { Yc } \\
\text { How War } \\
\text { Changed } \\
\text { Korean Food } \\
\text { Forever with } \\
\text { Maangchi \& } \\
\text { Japanese } \\
\text { Breakfast } \\
425\end{array}$ \\
\hline $\begin{array}{l}\text { Binging } \\
\text { with } \\
\text { Babish }\end{array}$ & N/A & $\begin{array}{c}\text { v=UvIkojfQDx } \\
\text { A } \\
\text { Binging with } \\
\text { Babish 1 } \\
\text { Million } \\
\text { Subscriber } \\
\text { Special: Taco } \\
\text { Town \& } \\
\text { Behind the } \\
\text { Scenes }\end{array}$ & $\begin{array}{c}\mathrm{v}=\_ \text {SObOsXrD } \\
\text { Rc } \\
\text { Pulled Pork | } \\
\text { Basics with } \\
\text { Babish } \\
1776\end{array}$ & $\begin{array}{c}\mathrm{v}=\mathrm{iF} 2 \mathrm{yGGfGv1} \\
8 \\
\text { Sous Vide | } \\
\text { Basics with } \\
\text { Babish Live } \\
222\end{array}$ & $\begin{array}{c}\text { v=nKKznGaN7 } \\
\text { PI } \\
\text { Tacos | Basics } \\
\text { with Babish } \\
\text { Live } \\
197\end{array}$ & $\begin{array}{c}\text { v=3S94NIJ80 } \\
\text { QA } \\
\text { Quesadillas | } \\
\text { Basics with } \\
\text { Babish Live } \\
156\end{array}$ \\
\hline
\end{tabular}

According to each channel video ID (e.g., V=23916491239) to mining the online commentary. Here are some example and outcome below:

\section{Fig 1. Example 1}

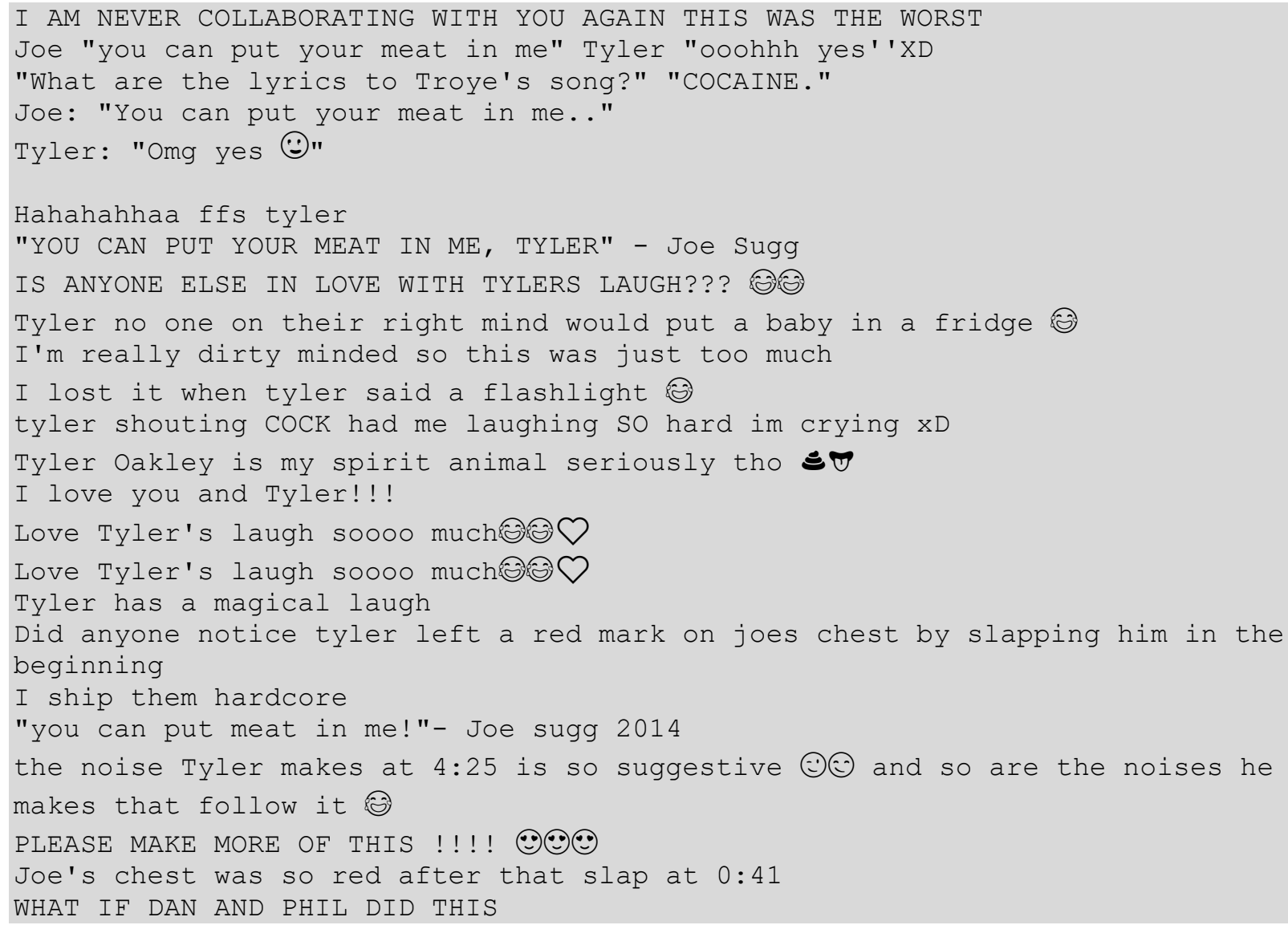

Fig 2. Outcome 1 
After I collected six categories of data, we used measured variables and the correlations between and measured variables and influence of viewers activity variables was conducted first. To exam the relationship between the measured independent variables in predicting emotional response, structural equation modelling was conducted.

\section{Result}

The research has three main findings, firstly, the hot topic keywords of public attention in different entertainment videos are slightly different, in addition, the public's positive emotions for Haul videos and unboxing videos with positive emotions increasing as whole. Public's comments are more positive and excited. Second, game video is the most popular category of video. Finally, the number of video views fluctuated greatly with the upload time, the number of viewers reached the peak in the week after the latest video uploaded in the first stage, in the second phase, the number of people dropped rapidly form the peak during a period, and third phase entered a slow decline period, until the end, the number of visitors is fixed at a constant value.

\section{Conclusion}

In the research, we analyzed different categories of video and proposed new method of social media comments to analyze the viewers' needs. This method uses gender as an example to analyze viewer preference. Through empirical analysis of different categories of video, it is found that female viewers prefer videos such as Haul videos, unboxing videos and beauty teaching (online teaching), while male viewers pay more attention to games and technology-related unboxing videos.

Comments affected by video content, communication technology and media environment. Among the above six categories of video, we conclude that there are a lot of comments on game videos, but the negative sentiment is very high. Because viewers cannot take part in the real game, thus, their experience of immersive gameplay cannot be obtained.

\section{References}

[1] Ghani, Norjihan Abdul, Suraya Hamid, Ibrahim Abaker Targio Hashem, and Ejaz Ahmed, "Social media big data analytics: A survey", Computers in Human Behavior, 2018.

[2] Sapountzi, Androniki, and Kostas E. Psannis, "Social networking data analysis tools \& challenges", Future Generation Computer Systems, Vol.86, pp.893-913, 2018.

[3] Tanev Hristo, Bruno Pouliquen, Vanni Zavarella \& Ralf Steinberger (forthcoming). Automatic Expansion of a Social Network Using Sentiment Analysis. In: Annals of Information Systems, Special Issue on Data Mining for Social Network Data. Uspensky, B. (1973). A Poetics of Composition. University of California Press, Berkeley, California.

[4] Mazer, J., Murphy, R. and Simonds, C. 2007. I'll see you on 'Facebook': The effects of computermediated teacher self-disclosure on student motivation, affective learning, and classroom climate. Communication Education, 56(1): 1-17.

[5] Selwyn, N. Screw blackboard... do it on Facebook!: An investigation of students' educational use of Facebook. Paper presented to the Pole 1.0 - Facebook social research symposium. November15. University of London. http://www.scribd.com/doc/513958/Facebook-seminar-paper-Selwyn (accessed September, 2008).

[6] Tufekci, Z. 2008. Grooming, gossip Facebook and Myspace. Information, Communication \& Society, 11 (4): 544-64.

[7] Wellens, J., Hooley, T., Meek, J. and Madge, C. Forthcoming. "Facebook". In social integration and differentiation.

[8] Lange, P. 2007. Publicly private and privately public: Social networking on YouTube. Journal of Computer-Mediated Communication, 13(1) http://jcmc.indiana.edu/vol13/issue1/lange.html (accessed December, 2008). 